\title{
703-THE INDIRECT ACTION OF IONS UPON AMINO ACID TRANSPORT SYSTEM L IN THE S37 CELL
}

\author{
GEORGE M. MARTIN, MARK S. ELLIOTT, JOHN P. MINTON, ROBERT ZAND and RICHARD \\ H. MATTHEWS \\ Departments of Physiological Chemistry and Surgery, The Ohio State University College of Medicine, \\ Columbus, $\mathrm{OH} 43210$ (U.S.A.) and Biophysics Research Division, Institute of Science and Technology, \\ University of Michigan, Ann Arbor, MI (U.S.A.)
}

(Manuscript received March 2nd 1984)

\section{SUMMARY}

The uptake into $\mathrm{S} 37$ ascites cells of an L-system specific analog, 2-amino-bicyclo-(3,2,1)-octanecarboxylic acid ( $A B O C A)$, was inconsistently inhibited by deletion of sodium ion from the incubation medium. We note that there have been conflicting reports from various other laboratories as to the effect of sodium ion on the transport of L-system specific analogs. The uptake of labeled exo-2-aminobicyclo-(2,2,1)heptane-2-carboxylic acid $(\mathrm{BCH})$ was also diminished by the removal of sodium from the medium. The $K_{m}$ values for these substrates were increased and $v_{\text {max }}$ values decreased as the sodium ion concentration was decreased or abolished. Transport behavior was also found to be affected by varying the medium potassium ion concentration with valinomycin present. The sodium effect was abolished by preincubation with cyanide and deoxyglucose. The results suggest an indirect effect of sodium ion upon transport system $L$ : system $L$ is energetically supported by a membrane potential.

\section{INTRODUCTION}

The consensus of several laboratories studying amino acid transport would be that amino acid transport system $L$ prefers amino acids having moderately large apolar side chains, such as leucine or exo-2-aminobicyclo-(2,2,1)-heptane-2-carboxylic acid (BCH). System $L$ is particularly effective in exchange processes. The question of a possible energetic support for system $L$ remained unclear. Inui and Christensen [1] suggested that system L was sodium independent. However, McClellan and Schafer [2] reported that the uptake of BCH was inhibited $60 \%$ by deletion of sodium ion from the medium. The uncertain question of a possible energetic support for system $\mathrm{L}$ and existing discrepancies in possible ion effects upon system L stimulated a more detailed investigation of ion effects on this transport system. We have found variable results in certain circumstances, which may help to explain the differences reported by other laboratories. There does appear to be an indirect effect of sodium ion upon transport system $L$. 


\section{EXPERIMENTAL}

General procedures for transport experiments were similar to those reported in detail elsewhere [3]. Optically active amino acids which were commercially available were obtained from Aldrich Chemical Company. Valinomycin was from Sigma Chemical Company. $\left[{ }^{14} \mathrm{C}\right]-\mathrm{BCH}$ was obtained from the laboratory of Dr. Margaret Neville. The synthesis of 2-aminobicyclo-(3,2,1)-octane-2-carboxylic acid (ABOCA) has been described [4]. Labeled compounds were checked for purity by radiochromatography. In experiments in which choline chloride replaced sodium, the concentrations of the cations were kept constant. In sodium-free media, potassium phosphate buffer replaced sodium phosphate buffer. Nine or ten replications of control or inhibited samples arranged alternately were employed in some experiments to facilitate statistical comparisons.

In the experiment in which the effect of a sodium gradient on $\mathrm{BCH}$ uptake was studied in poisoned cells, the S37 cells were incubated for $15 \mathrm{~min}$. at $20^{\circ} \mathrm{C}$ in $5 \mathrm{~m} \mathrm{M}$ $\mathrm{NaCN}, 5 \mathrm{~m} M$ deoxyglucose, and $0.1 \mathrm{~m} M \mathrm{BCH}$. The cells were then transferred to an environment containing $0.1 \mathrm{~m} M\left[{ }^{14} \mathrm{C}\right]-\mathrm{BCH}, 5 \mathrm{~m} M$ NaCN, $5 \mathrm{~m} M$ deoxyglucose, and $17 \mathrm{~m} \mathrm{M} \mathrm{NaCl}$ in one half the vials and no $\mathrm{NaCl}$ in the other half. Choline chloride replaced sodium chloride and potassium phosphate buffer replaced sodium phosphate buffer in the sodium-free media.

In an experiment designed to vary the membrane potential, S37 cells were incubated for $80 \mathrm{~min}$. in a medium containing $0.1 \mathrm{mM}\left[{ }^{14} \mathrm{C}\right]-\mathrm{BCH}, 6 \mathrm{~m} M$ potassium ion, $60 \mathrm{~m} M$ sodium ion, and no valinomycin (this was continued as the control medium). S37 cells were then transferred to a medium containing $60 \mathrm{~m} M$ sodium ion, $10 \mu M$ valinomycin, $0.1 \mathrm{~m} M\left({ }^{14} \mathrm{C}\right)-\mathrm{BCH}$, and varying concentrations of $\mathrm{K}+$ (compensated by choline).

\section{RESULTS}

\section{Labeled BCH uptake}

Uptake of $\left({ }^{14} \mathrm{C}\right)-\mathrm{BCH}$ was inhibited when sodium ion was deleted from the incubation medium (Table 1, Fig. 1). In an experiment in which a large number of replicates were performed at a single concentration, the conclusion that there was greater uptake with sodium ion present in the medium was arrived at with a high level of statistical significance (Table 1). Uptake of labeled BCH across a broad concentration range yielded concentrative uptake in the absence of sodium ion, but a larger $K_{m}$ and a decreased $v_{\max }$ (Fig. 1).

\section{Labeled $A B O C A$ as the test substrate}

Uptake of labeled ABOCA across a concentration range was on occasion markedly dependent upon the presence of sodium ion in the medium, but more often was affected little or none by the deletion of sodium ion from the medium. The variable 


\section{TABLE 1}

Effect of medium sodium ion on BCH uptake in $\mathbf{S} 37$ cells

$c_{i}$ is the intracellular concentration $(\mathrm{mM})$ of $\mathrm{BCH}$ after a 2 minute incubation at $20^{\circ} \mathrm{C}$ with $1 \mathrm{mM}$ $\left[{ }^{14} \mathrm{C}\right]-\mathrm{BCH}$ present in all media. Choline chloride replaced sodium chloride and potassium phosphate buffer replaced sodium phosphate buffer in the sodium free media. The two sets of values differ significantly at $p<0.005$ by the Wilcoxon ranking test [5]

\begin{tabular}{rl}
\hline$c_{i}$ with $\mathrm{Na}^{+}$ & $c_{i}$ without $\mathrm{Na}^{+}$ \\
\hline 2.65 & 2.23 \\
2.56 & 2.35 \\
2.61 & 2.27 \\
2.62 & 2.12 \\
2.59 & 2.06 \\
2.71 & 1.98 \\
2.56 & 2.09 \\
2.66 & 1.87 \\
2.66 & 2.19 \\
mean 2.62 & 2.13 \\
\hline
\end{tabular}

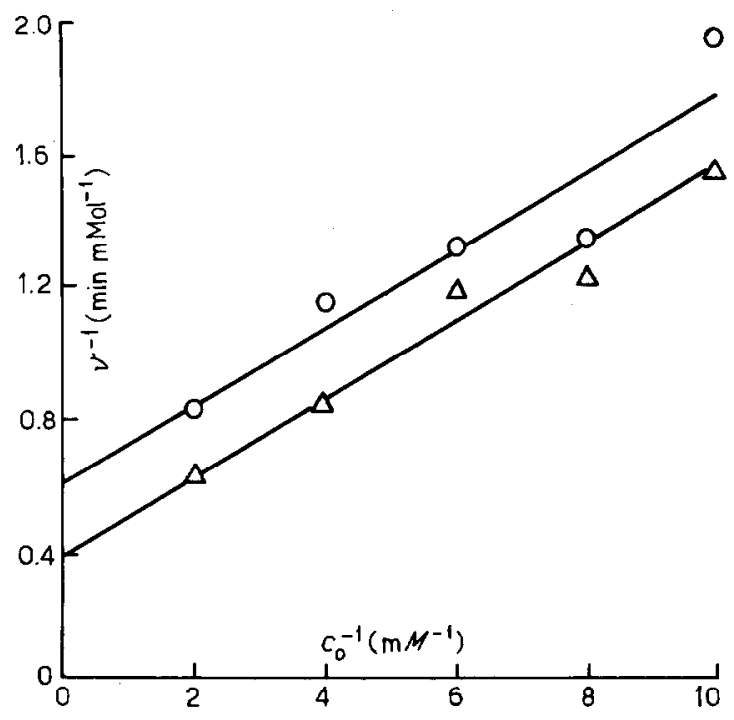

Fig. 1. Uptake of varying concentrations of $\left[{ }^{14} \mathrm{C}\right]-\mathrm{BCH}$ from media in which either no sodium was present $(O)$ or in which $20 \mathrm{mM}$ sodium ion was present $(\Delta)$. Data points are the averages of 2 samples, and are presented according to the method of Lineweaver and Burk [6]. $1 / c_{0}$ is the reciprocal of medium concentration.

results from this experiment are shown as Fig. 2. In another experiment in which multiple replicates at single concentrations were employed, labeled ABOCA uptake was more markedly sodium dependent (Table 2). An experiment with multiple replicates was also conducted with high concentrations of NMeAIB and homo- 


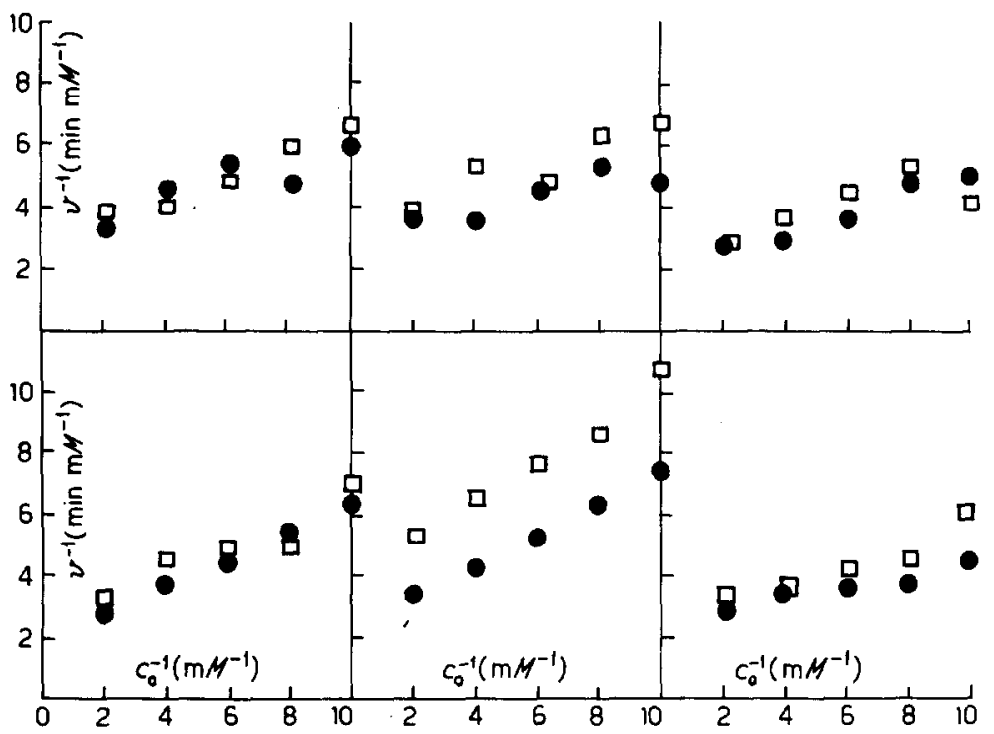

Fig. 2. Uptake of varying concentrations of $\left[{ }^{14} \mathrm{C}\right]$-ABOCA on several occasions. Media contained no sodium [ $\square$ ), or else $154 \mathrm{~m} M$ sodium ion (๑). Data are plotted according to Lineweaver and Burk [6]

TABLE 2

Effect of medium sodium ion on ABOCA uptake in $\mathrm{S} 37$ cells

Conditions were as for Table 1 except that $1 \mathrm{~m} M$ ABOCA was utilized instead of $1 \mathrm{~m} M \mathrm{BCH}$. The two sets of values differ significantly at $p<0.005$ by the Wilcoxon ranking test [5]

\begin{tabular}{cl}
\hline$c_{i}$ with $\mathrm{Na}^{+}$ & $c_{i}$ without $\mathrm{Na}^{+}$ \\
\hline 0.43 & 0.12 \\
0.39 & 0.19 \\
0.41 & 0.20 \\
0.37 & 0.24 \\
0.37 & 0.25 \\
0.38 & 0.22 \\
0.33 & 0.25 \\
0.37 & 0.20 \\
0.32 & 0.08 \\
0.36 & 0.09 \\
mean 0.37 & 0.18 \\
\hline
\end{tabular}

arginine present to block systems $\mathrm{A}$ and $\mathrm{Ly}+$, respectively. A marked sodium effect was again found to occur (Table 3).

\section{Sodium gradients in poisoned cells}

In cells pretreated with $\mathrm{NaCN}$ and deoxyglucose, the imposition of a sodium ion gradient did not significantly enhance the uptake of labeled $\mathrm{BCH}$ (Table 4). 
TABLE 3

Sodium effects on ABOCA uptake with transport systems $\Lambda$ and $\mathrm{Ly}^{+}$blocked

Conditions were as in Table 2 except that $10 \mathrm{~m} M$ homoarginine and $30 \mathrm{~m} M$ NMeAIB were present in all media. The two sets of values differ significantly at $p<0.005$ by the Wilcoxon ranking test [5]

\begin{tabular}{rl}
\hline$c_{i}$ with $\mathrm{Na}^{+}$ & $c_{i}$ without $\mathrm{Na}^{+}$ \\
\hline 1.91 & 1.65 \\
1.84 & 1.73 \\
1.89 & 1.44 \\
2.10 & 1.49 \\
2.23 & 1.28 \\
2.67 & 1.53 \\
2.56 & 0.93 \\
2.94 & 1.21 \\
2.75 & 1.54 \\
1.84 & \\
mean 2.27 & 1.42 \\
\hline
\end{tabular}

TABLE 4

Sodium effect on BCH uptake in poisoned cells

Conditions were as specified in Experimental section. The two sets of values do not differ significantly at $p<0.05$ by the Wilcoxon ranking test [5].

\begin{tabular}{cl}
\hline$c_{i}$ with $\mathrm{Na}^{+}$ & $c_{i}$ without $\mathrm{Na}^{+}$ \\
\hline 0.22 & 0.24 \\
0.24 & 0.21 \\
0.25 & 0.24 \\
0.25 & 0.25 \\
0.28 & 0.27 \\
0.09 & 0.06 \\
0.11 & 0.08 \\
0.09 & 0.09 \\
0.20 & 0.20 \\
0.19 & \\
mean 0.19 & 0.18 \\
\hline
\end{tabular}

\section{Membrane potential effect on transport}

The inclusion of valinomycin in incubation media with varying concentrations of potassium ion led to a systematic variation in the labeled $\mathrm{BCH}$ uptake, uptake being the greatest from media with lower concentrations of potassium ion (Fig. 3). At an extracellular potassium concentration of $50 \mathrm{mM}$, there was no appreciable difference from control (no valinomycin present, potassium ion $6 \mathrm{~m} \mathrm{M}$ ). 


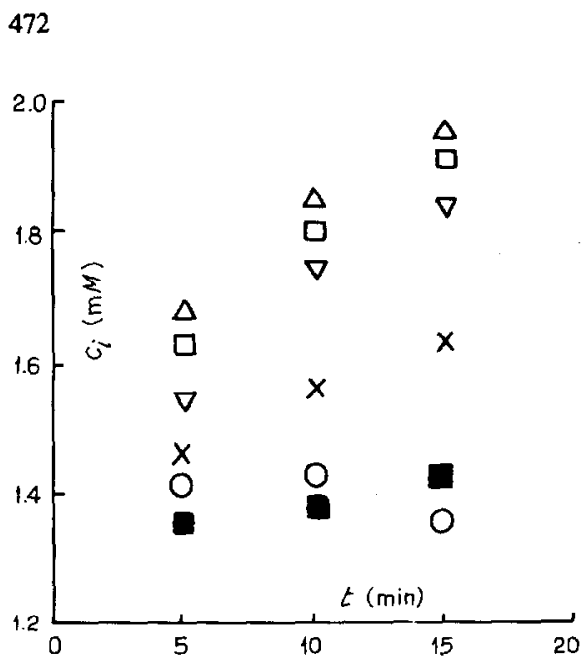

Fig. 3. Effect of varying the membrane potential on $\mathrm{BCH}$ uptake. Conditions were described in Experimental section. $c_{\mathrm{i}}$ is the intracellular concentration of labeled $\mathrm{BCH}$ following the incubation. Potassium concentrations with valinomycin present were $5 \mathrm{~m} M(\Delta), 10 \mathrm{~m} M(\square), 20 \mathrm{~m} M(\nabla), 30 \mathrm{~m} M(\times)$, $50 \mathrm{~m} M(\mathrm{G})$; the control incubation contained no valinomycin and $6 \mathrm{~m} M$ potassium ion (O).

\section{DISCUSSION}

The present study may help to explain certain differences in the literature regarding ion effects on transport system $\mathrm{L}$, and may add some further understanding as to the energetic basis of its operation.

We obtained quite variable results in one series of experiments with labeled ABOCA (Fig. 2, Tables 2 and 3). ABOCA was previously found to be specific for transport system $\mathrm{L}$ in the $\mathrm{S} 37$ cell, and with greater affinity for system $\mathrm{L}$ than that possessed by $\mathrm{BCH}$ [7]. ABOCA has not been widely available as a system L substrate, and we therefore also conducted an experiment in which systems $A$ and $\mathrm{Ly}^{+}$would be blocked (Table 3). This would suggest that endogenous factors could lead to different results on the question of ion dependence on a day to day basis. One implication of this finding is that differences seen between earlier reports $[1,2]$ may be explained as chance variation in the endogenous contents and conditions of cell preparations, or as due to variations in handling procedures from one laboratory to another. A second implication is that the ion effect seen on transport may be an indirect one, with some other factor interposed between the sodium ions and the transport system.

$\mathrm{BCH}$ was also selected as a substrate to utilize in this study in view of the prior agreement upon its specificity to transport system $\mathrm{L}$ in the Ehrlich and $\$ 37$ ascites tumor cells $[8,9]$. The uptake of $\mathrm{BCH}$ was significantly inhibited by the deletion of sodium ion from the medium (Table 1) and the inhibitions appeared to be based upon a decreased $v_{\max }$ as well as an increased $K_{m}$ (Fig. 1). The failure of a sodium gradient to stimulate BCH transport in poisoned cells (Table 4) would argue against a cotransport model for system L, of the type proposed for sugar transport in the 
intestine [10]. The variation of $\mathrm{BCH}$ transport with potassium ion concentration may be taken as a suggestion that the membrane potential is a significant energetic support for transport system $\mathbf{L}$. It is noteworthy that conditions which would induce a membrane potential intracellularly negative should not be expected to directly increase the uptake of zwitterionic amino acids with PI values of approximately 6. The inference to be suggested is that the effect of the membrane potential is on components of the carrier system in the membrane, causing a change in its properties which in turn causes favored release of the substrate amino acid on the intracellular side of the membrane.

\section{ACKNOWLEDGEMENT}

This work was supported in part by the Roessler Memorial Scholarship Fund.

\section{REFERENCES}

1 Y. Inui and H.N. Christensen, J. Gen. Physiol., 50 (1966) 203.

2 W. McClellan and R. Schafer, Biochim. Biophys. Acta, 311 (1973) 462.

3 R.H. Matthews and R. Zand, Biochemistry, 16 (1977) 3820.

4 R. Zand, O.Z. Sellinger, R. Water and R. Harris, J. Neurochem., 23 (1974) 1201.

5 K. Diem, (Editor), Documenta Geigy Scientific Tables, Geigy Pharmaceuticals, Ardsley, 1962, 6th ed., pp. 124-127, 191.

6 H. Lineweaver and D. Burk, J. Am. Chem. Soc., 56 (1934) 658.

7 M.S. Elliott, R.H. Matthews, J.P. Minton and R. Zand, Biochem., 20 (1981) 5105.

8 H.S. Tager and H.N. Christensen, J. Biol. Chem., 246 (1971) 7572.

9 R.H. Matthews, M. Sardovia, N.J. Lewis and R. Zand, Biochim. Biophys. Acta, 394 (1975) 182.

10 R.K. Crane, Fed. Proc., 24 (1965) 1000. 